\title{
The effects of lag and category membership on recognition memory in very young children
}

\author{
MARVIN W. DAEHLER and CAROLYN GRECO \\ University of Massachusetts, Amherst, Massachusetts 01003
}

\begin{abstract}
Children 13-30 months of age were briefly shown common objects on familiarization trials that later reappeared paired with new objects on test trials. The new objects were similar in shape, shared a common basic-level label, or were perceptually and conceptually unrelated to the familiarized stimuli. In the first of two experiments, children even as young as 13 months of age displayed significant preferences for novel stimuli in all test conditions. Length of lag between familiarization and test trial was not found to influence attentional preference. $A$ second experiment confirmed the findings of a significant novelty preference by 1-year-olds. In both experiments, older subjects displayed stronger preferences for novel over familiar stimuli when they were conceptually unrelated than when they were related. However, this greater preference was not consistently significant, as found in previous studies.
\end{abstract}

Very young children appear to have excellent visual recognition memory (for a review of the relevant literature, see Werner \& Perlmutter, 1979). Moreover, the procedures for investigating recognition memory have become useful for examining category knowledge in preverbal children. Faulkender, Wright, and Waldron (1974) found that for 3-year-olds, attention habituated during repeated presentations of pictures of animals, fruit, or environmental patterns. These subjects subsequently displayed greater recovery from habituation to novel extracategory pictures (pictures from a different category) than to novel intracategory pictures (pictures from the same category). In another study, Ross (1980) demonstrated that when even younger children, 12-, 18-, and 24-month-olds, were shown a series of men, animals, food, furniture, or individual letters of the alphabet, they also looked longer at novel extracategory stimuli than at novel intracategory exemplars.

While these findings provide support for the thesis that very young children possess fairly abstract conceptual knowledge, Daehler and O'Connor (1980) noted that the perceptual similarity of novel intracategory and extracategory stimuli to familiar stimuli may not be the same. The physical features of novel intracategory stimuli are likely to be more highly correlated with features of other category members than with the features of unrelated stimuli. Daehler and O'Connor (1980) showed a series of common objects to 13- to 34month-olds to examine the influence of shape similarity on looking preference. They used a continuous presenta-

This research was supported by Grant HD 09346 from the National Institute of Child Health and Human Development and Grant BNS 78-22970 from the National Science Foundation. The authors wish to thank Mary O'Connor Harper for her assistance in conducting Experiment 1. Requests for reprints should be sent to Marvin W. Daehler, Department of Psychology, University of Massachusetts, Amherst, Massachusetts 01003. tion procedure in which the lag between initial familiarization to a stimulus and its subsequent reappearance paired with a novel stimulus (test trial) ranged from 6 to 15 intervening trials. Three different types of test trials were included. On control trials, paired novel and familiar stimuli were perceptually and conceptually unrelated (e.g., key-scissors). On shape-similar trials, paired stimuli were again from different categories, but they were similar in gross perceptual shape (e.g., orangeball). On label-similar trials, paired novel and familiar stimuli were members of the same basic-level category; they were conceptually related and shared a common label but differed in size, color, and shape (e.g., two different varieties of flowers). When the data from all ages were considered, novel stimuli were found to be significantly preferred in all three conditions. More important, extracategory novel stimuli were attended to reliably more than intracategory novel stimuli and preferences for extracategory stimuli were equally strong in the control and shape-similar conditions. Category membership had a more influential effect on attentional preference than shape similarity had.

In a recent study, Scarborough (1977) found that under certain conditions perceptual similarity can affect recognition memory, at least in 4-, 8-, and 16-year-olds. Reaction times were longer for correct recognition of visually similar stimuli than for other stimuli, including those conceptually related, but only when the lag between initial presentation and test was relatively short (i.e., less than seven intervening pictures). These results suggest that the lag between familiarization and test may have been too long in the study conducted by Daehler and O'Connor (1980) to permit shape similarity to influence attention. Thus, one purpose of the present study was to examine the effects of short and long lag on preference for perceptually similar and dissimilar novel stimuli.

The present study also provided another opportunity to test the continuous recognition procedure with 
1-year-olds. Daehler and O'Connor (1980) found the youngest age group, 13-month-olds, failed to show a consistently significant preference for novel stimuli when a self-paced procedure for initiating trials was used. While older subjects quickly learned to press a plaque to see the stimuli on successive trials, the 13month-olds had considerable difficulty in acquiring this skill and the experimenter had to initiate trial changes for them. As a consequence, the attentional preference measure may not have been very sensitive for these subjects. The apparatus was modified in the present experiment to make it easier for 1-year-old subjects to learn to initiate trial changes. Perhaps they too would show recognition memory for large numbers of stimuli seen only briefly.

\section{EXPERIMENT 1}

\section{Method}

Subjects. Eight males and eight females, each at 13 (mean $=$ 12.7), $19($ mean $=19.2)$, and $30($ mean $=29.8)$ months of age, participated in this study. Four 13-month-olds and two 19-month-olds were replaced for failure to complete the experiment $(\mathrm{N}=5)$ or because of equipment malfunction $(\mathrm{N}=1)$. All subjects in the two oldest age groups were from the Amherst area and were tested at the university; subjects in the youngest group were from the Springfield area and were tested in a comparable room at the Child Study Center in Springfield.

Apparatus. The apparatus consisted of two adjacent windows $13.5 \times 15.3 \mathrm{~cm}$ in size and $6.5 \mathrm{~cm}$ apart. A one-way mirror $(20 \times 26 \mathrm{~cm}) 10 \mathrm{~cm}$ to the right of the windows was used to observe looking behavior. The windows and mirror were embedded in a curtain that served to partition the room. A response plaque, with a red button that could be illuminated, was located on the apparatus directly between the two windows. When the response plaque was pressed, electronic timers initiated a 6 -sec intertrial interval. During this interval, a screen lowered to conceal changes in stimuli occurring behind the windows. An event recorder was used to record trial onset, offset, and looking times.

Stimuli. The stimuli were 48 small common objects or toy replicas (see Daehler and O'Connor, 1980, for a complete list). The familiar and novel stimulus pairs in the shape condition differed in basic-level category membership and color but were similar in size and shape. In the label condition, the test pairs differed in size, shape, and color but shared the same label, as determined by pretesting a small group of 3-year-old children. Pairs of objects in the control condition shared no common features. A set of nine additional objects served as stimuli on training trials.

Design. There were six training trials, during which subjects learned to push the response plaque in order to see the next pair of stimuli, and 24 familiarization and 24 test trials. On familiarization trials, identical objects appeared in both windows. Test trials included a familiarization stimulus paired with a novel stimulus; each subject received eight test trials in each of the three test conditions. Stimuli for each condition were seen in every block of three familiarization trials with test trials interspersed at appropriate lags so that, in each condition, four trials were presented after a short lag ( 1 to 3 intervening trials) and four test trials were given after a long lag ( 9 to 11 intervening trials). Familiarization stimuli for each test pair were counterbalanced for each age and sex group. One of two different orders of presentation of the stimuli was given to half the subjects in each group.

Procedure. The subject was seated in a child-sized chair in front of the windows and to the right of the experimenter.
She/he was instructed to look in both windows and to press the button when she/he "wanted to see more things." At the beginning of each trial, the button became inoperable for $5 \mathrm{sec}$. At the end of this interval, the button was again illuminated to indicate another trial could be initiated at any time. All children learned to initiate trial changes during the six training trials and continued to press on all or nearly all subsequent trials.

Amount of time looking at each of the two windows during a trial served as the dependent measure. Interobserver reliability for recording looking times was established in an earlier experiment with adults as subjects and was found to range from .89 to .99 (Daehler \& Bukatko, 1977). All analyses of the data involving repeated measures were corrected according to the procedure suggested by Geisser and Greenhouse (1958) to reduce spurious significant effects due to heterogeneity of variance.

\section{Results}

Familiarization trials. The mean time attending to stimuli on familiarization trials for 13-, 19-, and 30month-olds was $2.16,2.59$, and $2.72 \mathrm{sec}$, respectively. A 3 (age) by 2 (sex) by 2 (familiarization set) by 3 (condition) by 8 (trials) by 2 (left vs. right window) repeated-measures analysis of variance performed on these data revealed no significant main effects or interactions for any of these factors.

Test trials. The mean number of seconds attending to familiar and novel stimuli for each age and condition is shown in Figure 1. Novel stimuli were attended to longer than familiar stimuli by all age groups and in all conditions, although the strength of attentional preference was clearly greater in some conditions than in others, especially for 19-month-olds. Looking times were entered into a 3 (age) by 2 (sex) by 3 (condition) by 2 (lag) by 4 (trials) by 2 (familiar vs. novel) repeatedmeasures analysis of variance. Reliable main effects were obtained for condition $[F(2,84)=12.06, p<.01]$ and novelty $[F(1,42)=97.09, p<.001]$, but these factors were also involved in a significant two-way interaction $[F(1,84)=5.02, p<.05]$. While novel stimuli were preferred over familiar stimuli in all three test conditions, the preference was considerably greater in the shape-similar and, to a lesser extent, the control conditions than in the label-similar condition. Age was not found to reliably interact with these two factors, but it is apparent from examination of Figure 1 that the data from the 19-month-olds primarily contributed to the interaction between condition and novelty. Subsequent analyses of variance carried out at each age level yielded a reliable interaction between condition and novelty for 19-month-olds only.

A significant main effect was also obtained for lag $[F(1,42)=4.75, p<.05]$. On test trials, stimuli were attended to less after a short lag than after a long lag (2.63 and $2.86 \mathrm{sec}$, respectively). However, lag did not significantly interact with condition or condition and novelty as the findings of Scarborough (1977) would suggest.

One final significant interaction involved age and condition $[F(1,84)=4.53, p<.05]$. Thirteen-montholds attended to items in the control condition least, 

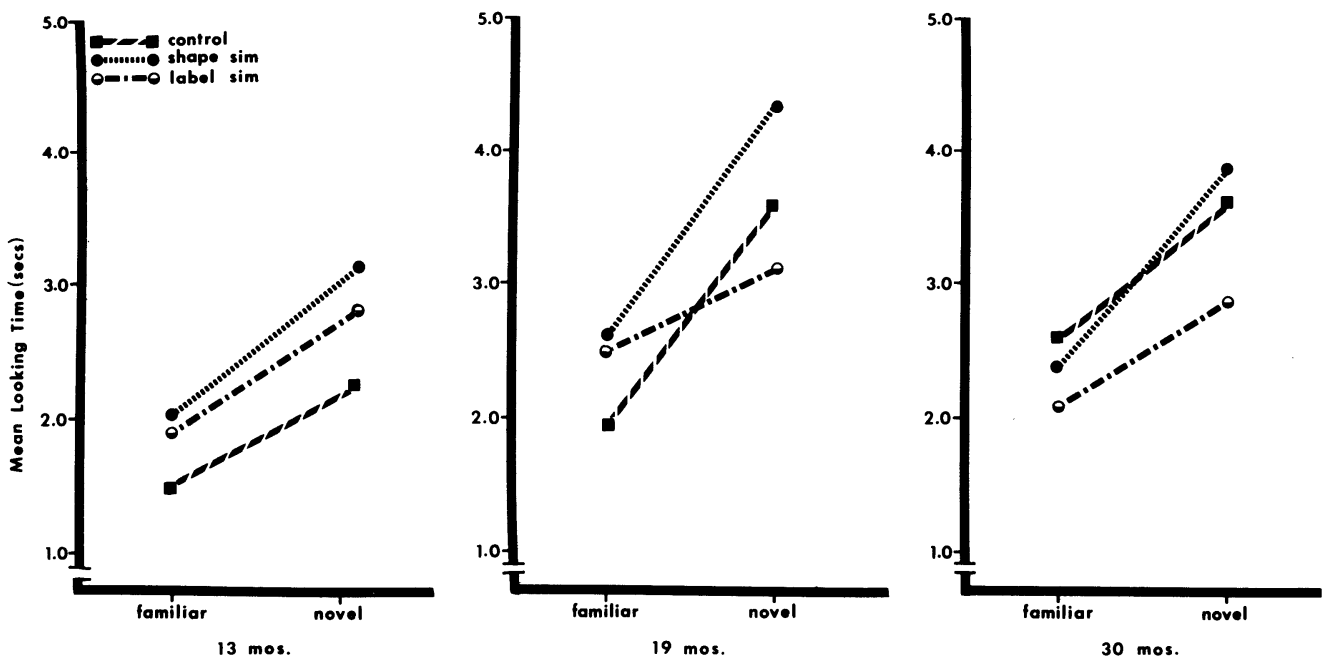

Figure 1. Mean number of seconds attending to familiar and novel stimuli (Experiment 1).

whereas 30-month-olds attended to stimuli in the label-similar condition least. Why this interaction emerged is unclear. Of more immediate importance was the finding that 13-month-olds preferred novel stimuli over familiar stimuli in all three test conditions $(p<.01$ or better).

\section{EXPERIMENT 2}

Even with a short lag, the data from this first experiment were consistent with those obtained by Daehler and O'Connor (1980) in failing to show that similarity in shape has a measurable influence on preference for novel extracategory stimuli. Perceptual similarity may affect reaction times of older children (Scarborough, 1977), but this does not appear to be a major factor in accounting for why novel intracategory stimuli are less preferred than novel extracategory stimuli (Faulkender et al., 1974; Ross, 1980). The results did reveal, however, that children as young as 13 months of age are able to recognize large numbers of stimuli seen only briefly. A minor alteration of the apparatus was sufficient to permit subjects in this age group to learn to initiate trial changes themselves. There was no indication, however, of greater preferences for novel extracategory than for novel intracategory stimuli by this age group.

Since the 13-month-old children and the older subjects came from different populations, a second experiment was carried out using 1- and 2-year-olds from the same population. The design of the experiment was also modified. Only label-similarity and control conditions were tested to permit counterbalancing of exemplars across conditions. Such counterbalancing had not been possible in previous experiments because of the constraints of finding different basic-level exemplars with similar shapes and novel intracategory exemplars dissimilar in shape.

\section{Method}

Subjects. Nine girls and nine boys, each at 14 (mean $=13.8)$ and 24 (mean $=23.7$ ) months of age, participated in this experiment. One 14-month-old was replaced because of experimenter error. All subjects were from the Springfield area.

Apparatus and Stimuli. The apparatus was identical to that used in Experiment 1. The stimuli were 40 common objects or toy replicas, some used in the previous experiment and some new ones. The familiar and novel stimuli in the label condition differed in size, shape, and color but shared the same common label, as determined by pretesting a small group of three-yearold children. Pairs of objects in the control condition shared no common features. An additional 12 objects were used on training and filler trials.

Procedure. The procedure was similar to that used in Experiment 1 . However, subjects were shown only 48 pairs of stimuli (6 training, 20 familiarization, 20 test, and 2 filler trials).

Subjects received 10 test trials in each of two conditions. Three different orders of presentation of stimuli were used so that stimuli served equally often as familiar and novel exemplars in the label and control conditions. Length of lag between familiarization and test varied randomly between six and nine trials.

\section{Results}

Familiarization trials. The mean time attending to stimuli on familiarization trials for 14- and 24-montholds was 2.41 and $2.70 \mathrm{sec}$, respectively. A 2 (age) by 2 (sex) by 3 (order) by 2 (condition) by 10 (trial) by 2 (left vs. right window) repeated-measures analysis of variance revealed no significant main effects or interactions.

Test trials. Figure 2 illustrates the mean looking times of familiar and novel stimuli in the control and labelsimilar conditions for each age group. Novel stimuli were again attended to more than familiar stimuli in all conditions. The data were entered into a 2 (age) by 2 (sex) by 3 (order) by 2 (condition) by 10 (trial) by 2 (familiar vs. novel) repeated-measures analysis of variance. A significant main effect for novelty $[F(1,24)=61.47$, $\mathrm{p}<.01]$ and a significant interaction between age and novelty $[F(1,24)=7.44, p<.05]$ were found. Older 

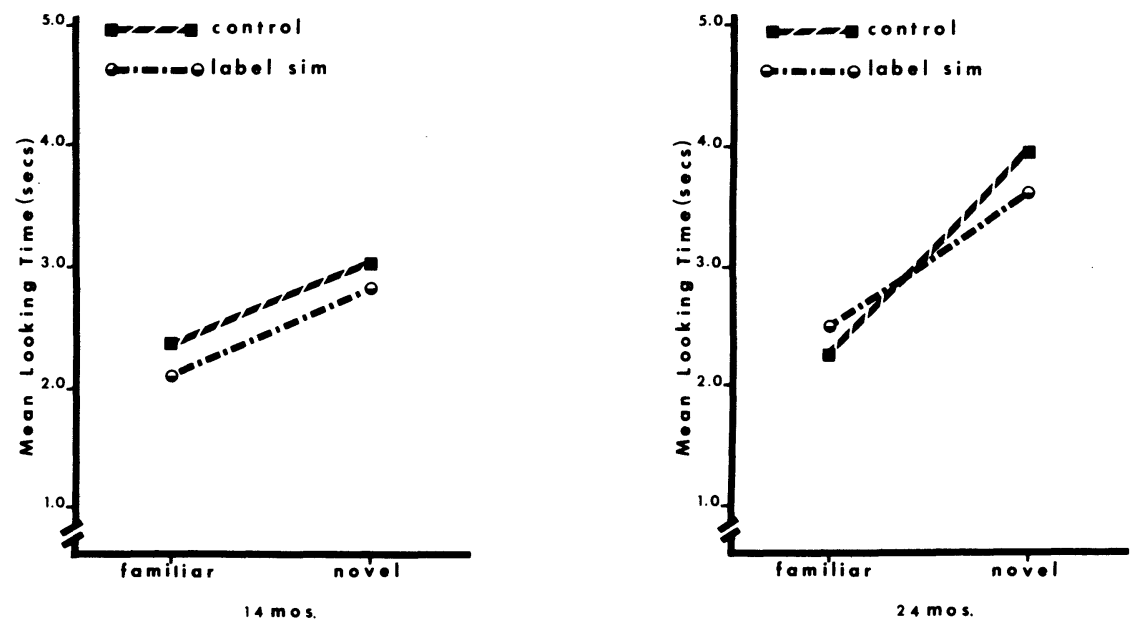

Figure 2. Mean number of seconds attending to familiar and novel stimuli (Experiment 2.

subjects not only looked at the stimuli longer than younger subjects did but, in addition, preferred novel over familiar stimuli relatively more. Analysis of cell means, however, did indicate that novel stimuli were attended to significantly longer than familiar stimuli by the 14-month-olds in both conditions. Once again, the data from only the older subjects suggested a greater preference for novel stimuli over familiar in the control condition than in the label-similar condition. However, the interaction involving age, condition, and novelty failed to be significant.

The only other reliable effect obtained in this analysis was a significant interaction between age and sex. While the mean time looking at stimuli on test trials by 24-month-old boys and both 14-month-old boys and girls were quite similar $(2.50,2.57$, and $2.51 \mathrm{sec}$, respectively), 24-month-old girls attended to the stimuli considerably longer $(3.55 \mathrm{sec})$. Exactly the same sex effect was found for this age group in another experiment (Daehler \& O'Connor, 1980), indicating that this may be a period when the looking behaviors of boys and girls are not comparable.

\section{DISCUSSION}

The results of the present experiments indicate that looking times can also be employed to investigate the 1-year-old's recognition memory for relatively large numbers of stimuli seen only briefly. While the preference for novel over familiar stimuli may not be as great in 1-year-olds as in somewhat older subjects, it is reliable and consistent for various test conditions. One-year-olds, however, did not display a comparatively stronger preference for novel extracategory than for novel intracategory stimuli, a finding that contrasts with those obtained by Ross (1980) for 1-year-olds and Daehler and O'Connor (1980) for older subjects. Ross presented subjects with a number of exemplars of a single category before testing and novel intracategory and extracategory stimuli were paired together on test trials. Direct comparison combined with extensive exposure to category members may provide a more sensitive measure of looking preferences for intra- and extracategory stimuli.

Older subjects in the present study failed to show consistently reliable preferences for novel extracategory over novel intracategory stimuli. The patterns of preferences for the 30-month-olds (Experiment 1) and 24-month-olds (Experiment 2) were in the expected direction, but the results were significant for only the 19-month-olds. The results obtained from the first experiment do, however, provide support for the conclusion that similarity in shape has very little influence on looking preferences for novel extracategory stimuli.

\section{REFERENCES}

Daehler, M. W., \& Bukatko, D. Recognition memory for pictures in very young children: Evidence from attentional preferences using a continuous presentation procedure. Child Development, 1977, 48, 693-696.

Daehler, M. W., \& O'Connor, M. P. Recognition memory for objects in very young children: The effect of shape and label similarity on preference for novel stimuli. Journal of Experimental Child Psychology, 1980, 29, 306-321.

Faulkender, P. F., Wright, J. C., \& Waldron, A. Generalized habituation of concept stimuli in toddlers. Child Development, 1974, 45, 1002-1010.

Geisser, S., \& Greenhouse, S. W. An extension of Box's results on the use of the F-distribution of multivariate analysis. Annals of Mathematical Statistics, 1958, 29, 885-891.

Ross, G. S. Categorization in 1- to 2-year-olds. Developmental Psychology, 1980, 16, 391-396.

Scarborough, H. S. Development of visual, name, and conceptual memory codes for pictures. Journal of Experimental Child Psychology, 1977, 24, 260-278.

Werner, J. S., \& Perlmutter, M. Development of visual memory in infants. In H. W. Reese \& L. P. Lipsitt (Eds.), Advances in child development and behavior (Vol. 14). New York: Academic Press, 1979.

(Received for publication October 16, 1981.) 